\title{
Efficacy and Safety of Topical Cyclosporine in Dry Eye Subjects Who Engage in Electronic Visual Tasking-The EMPOWER Study
}

\author{
Karl Stonecipher, ${ }^{1}$ Gail Torkildsen, ${ }^{2}$ Eugene McLaurin,${ }^{3}$ Linda Villanueva, ${ }^{4}$ George Ousler, ${ }^{5}$ David A Hollander ${ }^{5}$ \\ 1. TLC Laser Eye Centers, Greensboro, NC, USA; 2. Andover Eye Associates Inc., Andover, MA, USA; 3. Total Eye Care, P.A., Memphis, TN, USA; \\ 4. Carl Zeiss Meditec Inc, Dublin, CA, USA; 5. Ora Inc., Andover, MA, USA
}

DOI: https://doi.org/10.17925/USOR.2019.12.2.88

ntroduction: Dry eye is becoming more prevalent in younger individuals ( $<50$ years), partly owing to prolonged electronic visual tasking. This study assessed the effect of cyclosporine ophthalmic emulsion 0.05\% (COE; Restasis ${ }^{\circledR}$, Allergan plc, Dublin, Ireland) on dry eye subjects who experience ocular discomfort during electronic visual tasking. Methods: Open-label study in dry eye subjects using electronic devices daily with ocular discomfort (Ora Calibra ${ }^{\mathrm{TM}}$ Ocular Discomfort and 4-Symptom Questionnaire score $\geq 2$ ) and ocular surface staining. Subjects were administered COE twice daily for 6 months; change from baseline in ocular surface staining, reading performance, subject-rated visual function, ocular symptoms, and tear production were assessed. Results: Forty-six of 51 subjects completed the study. At 6 months, significant improvements were observed in total ocular surface and central corneal staining ( $p \leq 0.0001)$ and visual function measured by Ocular Surface Disease Index ${ }^{\odot}$ (OSDI) questions (blurred vision, poor vision, night driving, working with a computer, and watching television [all $\left.p \leq 0.0160\right]$ ). No significant differences were observed in reading performance. Schirmer's score increased by $4.0 \pm 7.1 \mathrm{~mm}$ ( $p=0.0004)$; $32 \%$ of subjects had increases $\geq 5 \mathrm{~mm}$. Percentage of subjects with OSDI scores $>32$ (severe) was reduced from $76 \%$ at baseline to $44 \%$. Conclusion: After 6 months of COE, ocular surface staining and subject-rated visual function were significantly improved.

\section{Keywords}

Dry eye disease, cyclosporine ophthalmic emulsion, ocular surface staining, visual function, video display terminals, electronic visual tasking

Disclosures: Karl Stonecipher is a consultant to and a speaker for Allergan plc. Gail Torkildsen is a consultant to Allergan plc and Ora, Inc. Eugene McLaurin has received research support from Aciex, Acucela, Alcon, Allergan plc, AstraZeneca, Bausch \& Lomb, Inotek Pharmaceuticals, InSite Vision, and Lexicon Pharmaceuticals. Linda Villanueva was an employee of Allergan plc when the study was conducted and is currently an employee of Carl Zeiss Meditec Inc. George Ousler is an employee of Ora Inc. David A Hollander was an employee of Allergan plc at the time the study was conducted and is currently an employee of Ora, Inc.

Acknowledgments: The authors would like to thank the subjects who participated in this study Medical writing and editorial assistance were provided to the authors by Kakuri M Omari, PhD, CMPP, and Stuart Murray, MSc, CMPP, of Evidence Scientific Solutions, Inc., Philadelphia, PA, and funded by Allergan plc, Dublin, Ireland. No honoraria or payments were made for authorship.

Compliance with Ethics: This study was performed in compliance with the ethical principles of the Declaration of Helsinki and Good Clinical Practice, and the Health Insurance Portability and Accountability Act. The study protocol was reviewed by the Alpha Independent Review Board, San Clemente, California. All subjects provided written consent before initiating any study treatment.

Data Sharing Statement: Data reported in this manuscript are available within the article and/or its supplementary materials. Allergan will share de-identified patientlevel data and/or study-level data, including protocols and clinical study reports, for phase II-IV trials completed after 2008 that are registered on ClinicalTrials.gov or EudraCT. The indication studied in the trial must have regulatory approval in the United States and/or the European Union, and the primary manuscript from the trial must be published prior to data sharing. To request access to the data, the researcher must sign a data use agreement. All shared data are to be used for noncommercial purposes only. More information can be found on http://www.allerganclinicaltrials.com/

Authorship: The named authors meet the International Committee of Medical Journa Editors (ICMJE) criteria for authorship of this manuscript, take responsibility for the integrity of the work as a whole, and have given final approval for the version to be published.

Received: May 28, 2019 Accepted: November 20, 2019

Citation: US Ophthalmic Review. 2019;12(2):88-95

Corresponding Author: Karl Stonecipher, Medical Director, TLC Laser Eye Centers 1002 North Church Street, Greensboro, NC 27408, USA. E: stonenc@aol.com

Support: The work was supported by Allergan plc, Dublin, Ireland. Allergan participated in the design of the trial, data management, analyses and interpretation, and preparation, review and approval of the manuscript.

\section{Introduction}

In the recently updated Dry Eye WorkShop DEWS II report, dry eye is defined as a multifactorial disease of the ocular surface characterized by loss of homeostasis of the tear film, accompanied by ocular symptoms, tear film instability, hyperosmolarity, ocular surface inflammation and damage, and neurosensory abnormalities. ${ }^{1}$ Chronic, T cell-mediated inflammation of the ocular surface and lacrimal glands plays a prominent role as signaling pathways, activated in a variety of cell types by stress to the ocular surface, upregulate proinflammatory proteins, contribute to further damage, and lead to a self-perpetuating inflammatory cycle. ${ }^{2-4}$ Dry eye is often associated with ocular discomfort, which typically worsens in the evening. In addition, intermittent blurred vision often develops from corneal surface irregularities and tear film abnormalities though normal visual acuity may be measured on a standard eye chart. ${ }^{1,6}$ Impaired visual function can significantly impact visiondependent activities associated with social and physical functioning (e.g., reading, driving at night) and has an adverse impact on workplace productivity and overall quality of life.-9 Patients with severe dry eye have reported reduced quality of life comparable to that of moderate to severe angina. ${ }^{10}$

Dry eye disease is a global public health concern. Prevalence increases with age and current estimates suggest that $5-50 \%$ of the global population is affected by dry eye. ${ }^{11}$ In recent years, the prevalence may have increased as a result of the aging population and more frequent use of contact lenses, computers, smartphones, and tablets. ${ }^{12-14}$ In a recent meta-analysis of prior reports of dry eye disease prevalence in visual display terminal workers, the prevalence of dry eye ranged from 9.5-87.5\% across 16 studies, with the vast differences attributed to differences in criteria for defining dry eye. ${ }^{15}$

Symptomatic dry eye disease can present initially without evidence of ocular surface damage or changes in tear production.7.8,16 Discordance between 
diagnostic tests and patient-reported symptoms ${ }^{17,18}$ poses a challenge for accurately diagnosing and treating patients with dry eye. Several therapeutic options are currently available in the United States to patients with dry eye. Cyclosporine ophthalmic emulsion 0.05\% (Restasis ${ }^{\circledast}$, Allergan plc, Dublin, Ireland) was approved in 2002 to increase tear production in patients whose tear production is presumed to be suppressed owing to ocular inflammation associated with keratoconjunctivitis sicca. ${ }^{19}$ In clinical trials, cyclosporine ophthalmic emulsion resulted in greater improvements in tear production, corneal staining, and dry eye symptoms compared with vehicle. ${ }^{20,21}$ In 2016, lifitegrast ophthalmic solution 5\% (Xiidra ${ }^{\circledR}$, Novartis International AG, Basel, Switzerland), was approved for the treatment of the signs and symptoms of dry eye disease. ${ }^{22}$

Given the increasing use of visual display terminals and their impact on dry eye, the present study was conducted to evaluate the efficacy of cyclosporine ophthalmic emulsion $0.05 \%$ in dry eye subjects with associated ocular discomfort when engaging in frequent electronic visual tasking.

\section{Methods}

\section{Study design and eligibility}

This multicenter, open-label study (ClinicalTrials.gov identifier NCT02554981) was performed in compliance with the ethical principles of the Declaration of Helsinki and Good Clinical Practice, Health Insurance Portability and Accountability Act. The study protocol was reviewed by the Alpha Independent Review Board, San Clemente, California. All subjects provided written consent before initiating any study treatment.

Subjects were eligible if they were between $18-55$ years of age with a history of a dry eye disease in both eyes and dry eye symptoms ( $\geq 2$ on the Ora Calibra'⿳⺈ Ocular Discomfort and 4-Symptom Questionnaire) when using video display terminals. Key inclusion criteria also included ocular surface staining ( $\geq 2$ and $<4$ in at least one corneal region, central corneal staining $\geq 2$ on the Ora Calibra scale), conjunctival redness ( $\geq 1$ on the Ora Calibra scale), and Schirmer score $<10 \mathrm{~mm}$ in at least one eye, and total Ocular Surface Disease Index ${ }^{\oplus}\left(\left.\mathrm{OSD}\right|^{\circ}\right)>12$. A diagnosis of Sjögren's syndrome was not required for inclusion. Key exclusion criteria included active ocular infection, use of Restasis ${ }^{\circledR}$ within the prior 90 days, refractive surgery within 12 months, punctal occlusion, use of contact lenses, or use of systemic medication known to cause ocular drying that had not been used on a stable dosing regimen for at least 30 days and was expected to remain stable during the study period.

\section{Study visits and interventions}

The study consisted of five visits: visit 1 (day 0), visit 2A (day $0 \pm 1-3$ days, morning), visit $2 B(10 \pm 2$ hours following visit $2 A)$, visit 3 (1 month after study initiation), visit $4 \mathrm{~A}$ (6 months after study initiation), visit $4 \mathrm{~B}$ (10 \pm 2 hours following visit 4A), and visit 5 (exit visit; $1-3$ days after visit 4). Additional telephone contact was made between visits at day 14 and months 2, 3, 4, and 5 after study initiation. Subjects were queried about the average amount of time per day during the last week spent using a computer, television, or smartphone or playing a videogame. At visit 2B, subjects received a 6-month supply of cyclosporine ophthalmic emulsion $0.05 \%$ (Restasis ${ }^{\circledR}$ ) and were instructed to instill one drop of the emulsion twice daily in both eyes for the duration of the study, beginning the evening of visit 2B. Artificial tears (Refresh Optive ${ }^{\circledR}$ Advanced, Allergan plc, Dublin, Ireland) were also provided for subjects to use as needed. At visits $2 \mathrm{~A}$ and $4 \mathrm{~A}$, a diurnal blink device (Ora, Inc.), which is a validated electromyographic-based blink monitor, was fitted. ${ }^{23}$ This device monitors blink pattern and rate over a period of approximately 10 hours. During this period, each subject recorded electronic visual tasking using a daily diary to note activity at 30-minute intervals. Diaries were collected at visits 2B and 4B and the blink device was removed.

\section{Primary efficacy assessments}

The primary efficacy measures were ocular surface staining and visual function at month 6. Ocular surface staining was assessed by instilling $5 \mu \mathrm{L}$ sodium fluorescein $2 \%$ solution and $10 \mu \mathrm{L}$ lissamine green solution into the inferior conjunctival cul-de-sac of each of the subject's eyes. Fluorescein staining was evaluated 3 to 5 minutes after instilling fluorescein, which allowed maximum fluorescence to be achieved, while lissamine green staining was evaluated 30 seconds after blinking following instillation of lissamine green; eyes were graded using a 5-point Ora Calibra Corneal and Conjunctival Staining Scale (0 [none] to 4 [confluent staining, denoted as severe], where half unit increments were permitted), respectively. ${ }^{24,25}$ Both vital dyes were used to assess both corneal and conjunctival staining: ocular surface staining was assessed as total grade (all corneal and conjunctival regions), total cornea grade (central, inferior, and superior regions), total conjunctiva grade (temporal and nasal regions), and each of the five individual regions.

Visual function was evaluated using a series of three separate tests-the Ora Calibra Menu Reading Test, IReST Reading Test, and Wilkins Rate of Reading Test. These tests evaluated the time taken to read a passage, the number of words read incorrectly, and font size.

\section{Secondary efficacy assessments}

Secondary efficacy measures of visual function and symptom assessments included tear film break-up time (TFBUT), interblink interval (IBI), Ocular Protection Index 2.0 (OPI 2.0), the Ora Calibra Ocular Discomfort Scale, the Ora Calibra Ocular Discomfort and 4-Symptom Questionnaire, and tear production assessed by Schirmer's test score.

Subject-rated visual function was evaluated using the OSDI questionnaire (Appendix 1), which consists of 12 questions that assess ocular symptoms (light sensitivity, grittiness, pain/soreness), ability to perform visualrelated tasks (blurry vision, poor vision, reading, night driving, computer/ bank machine use, watching television), and the effect of environmental conditions (wind, low humidity, air conditioning). These were graded on a scale ranging from 0 (none of the time) to 4 (all of the time), and the total score was calculated as OSDI = [(sum of all scores for questions answered) $x$ 100] / [(number of questions answered) $x$ 4]. ${ }^{10,26}$ The total OSDI scores were further categorized according to severity (mild $>12$, moderate $>22$, and severe $>32$ ), and the percentage of subjects was assessed in each category at baseline and after 6 months. ${ }^{27}$

The change in TFBUT from baseline to visit 5 (6 months) was assessed in the worse eye (at baseline). The mean change in IBI from baseline to visit 5 was assessed by measuring the time (seconds) between blinks in the worse eye using the blink device. An increase from baseline in the IBI indicates a worsening in visual function (more frequent blinks). The OPI 2.0 system implements fully automated software algorithms that provide a real-time measurement of corneal exposure (break-up area) for each $\mathrm{IBI}$ during a 1-minute video and was assessed at baseline and visit $5 .{ }^{28}$

Subjects were asked to subjectively grade their ocular discomfort for each eye using a 5-point Ora Calibra Ocular Discomfort Scale (0 [no discomfort] 
to 4 [severe]). The severity of dry eye symptoms was assessed using the 6-point Ora Calibra Ocular Discomfort and 4-Symptom Questionnaire; five ocular symptoms, overall discomfort, burning, dryness, grittiness, and stinging, were graded on a scale from 0 (no pain) to 5 (worst pain). ${ }^{24}$ As part of the visual function assessments, ocular discomfort scores were also collected following Wilkins Rate of Reading Test. In addition, diurnal dry eye symptoms were assessed in the morning and evening on study days that blink rate was measured (visit 2 and visit 4).

Schirmer's test (without anesthesia) was performed to measure tear production. Sterile Schirmer's test strips (Tear Flo, Rose Stone Enterprises, Alta Loma, CA, USA) were placed in the lower cul-de-sac at the junction of the temporal and central one-third of the lower eyelid margin. Subjects were instructed to close their eyes for 5 minutes, then the strip was removed, and the length of the moistened area measured.

\section{Safety assessments}

Safety measures included monitoring adverse events (AEs), slit lamp biomicroscopy, and visual acuity. The Early Treatment of Diabetic Retinopathy Study chart was used to assess visual acuity. ${ }^{29}$ An increase of $\geq 0.22$ in LogMAR score was judged as clinically significant and recorded as an AE.

\section{Statistical analyses}

For all analyses, the worse eye and fellow eye were identified for each subject. The worse eye was defined as the eye with the highest central corneal fluorescein staining at baseline (visit 1). If both eyes had similar central staining at baseline, the right eye was considered the worse eye for analysis. Efficacy analyses were performed on the intent-to-treat (ITT) population (all subjects receiving one dose of study drug) with observed data only by worse eye. The average of both eyes was used as a secondary analysis. The per-protocol population included subjects who completed the study without any major protocol deviations. The safety population was the same as the ITT population.

The primary efficacy endpoints were the change in fluorescein staining and lissamine green staining as graded by the Ora Calibra scale, and change in the Ora Calibra Menu Reading Test, IReST Reading Test, and Wilkins Rate of Reading Test at visit 5 (6 months) compared with baseline. Secondary efficacy analyses included OSDI individual questions and overall score, the change in TFBUT, blink rate (IBI), OPI 2.0, Ora Calibra Ocular Discomfort Scale, Ora Calibra Ocular Discomfort and 4-Symptom Questionnaire, and Schirmer's test score at visit 5 compared with baseline. Quantitative variables were summarized using number of observations (n), mean, standard deviation (SD), median, minimum, and maximum. For data collected over time, the observed data at each visit were summarized and change from baseline (visit 1) was summarized at visit 5. Safety measures were summarized using counts and percentages. Paired $t$-tests were used to determine whether changes from baseline indicated any significant improvements for all efficacy analyses, with $p<0.05$ level of significance. Statistical programming and analyses were performed using SAS ${ }^{\circledR}$ Version 9.2 (Cary, NC, USA). Data and associated protocols are available upon request to Allergan plc.

\section{Results}

A total of 51 subjects were enrolled between July 21, 2015, and March 24, 2016, in two centers in the United States; one subject discontinued the study before dosing. The mean \pm SD age was $46.3 \pm 8.3$ years and the majority of subjects were female (82\%) and white (72\%). Overall, 46 (90\%) subjects completed the study (per-protocol population). Three subjects withdrew consent, one was discontinued due to an AE unrelated to study drug, and one subject was withdrawn for administrative reasons.

\section{Efficacy outcomes}

At baseline, mean \pm SD total ocular surface fluorescein staining was $11.6 \pm 2.0$ in the worse eye and $11.2 \pm 2.2$ in the fellow eye. Significant reductions in fluorescein staining were observed in total ocular surface areas of both the worse eye $(-2.1 \pm 2.4 ; p<0.0001)$ and fellow eye $(-1.6 \pm$ 2.6; $p=0.0001$ ) after 6 months of topical cyclosporine (Ora Calibra scale; Figures $1 A$ and $B$ ). In addition, significant reductions were also observed after 6 months of cyclosporine in fluorescein staining in total cornea $(-1.5 \pm 1.5$ $[p<0.0001],-1.0 \pm 1.8[p=0.0003])$, central cornea $(-0.8 \pm 0.7[p<0.0001],-0.5 \pm$ $0.8[p=0.0005])$, inferior cornea $(-0.4 \pm 0.8[p=0.0008],-0.3 \pm 0.8[p=0.0057])$, and total conjunctiva $(-0.6 \pm 1.2[p=0.0017],-0.6 \pm 1.2[p=0.0011])$ for both the worse and fellow eye, respectively. The magnitude of reduction in staining was smaller with lissamine green than with fluorescein, though statistically significant reductions in lissamine green staining were observed for central cornea in the worse eye as well as total conjunctiva, total ocular surface area, inferior cornea and temporal cornea in the fellow eye (Figures $1 C$ and D).

At baseline, reading test results (Ora Calibra Menu, IReST, and Wilkins Rate) varied depending on the type of test but overall suggested a high degree of reading competency in terms of the mean \pm SD time to read a passage $(42.4 \pm 18.4,54.3 \pm 13.6$ and $126.0 \pm 28.6$ seconds, respectively), and the number of words read incorrectly $(1.6 \pm 2.8,3.2 \pm 5.5$, and $16.6 \pm 22.0$, respectively). After the 6-month treatment period, the time to read a passage decreased modestly when assessed with the Ora Calibra Menu Reading Test ( $-3.0 \pm 14.8$ seconds), the IReST Reading Test ( $-1.2 \pm 8.4$ seconds) and the Wilkins Rate of Reading Test $(-0.7 \pm 24.5$ seconds), though changes from baseline were not statistically significant (Table 1).

Changes in subject-rated visual function were also assessed following treatment with topical cyclosporine using the OSDI questionnaire. After 6 months of treatment, significant reductions (improvement) were observed in all visual-related questions except for reading (Figure 2). Significant reductions were also observed for the questions related to ocular symptoms and the effect of the environment on dry eye symptoms (Figure 2). Overall, the total OSDI score significantly decreased from $45.1 \pm 17.8$ at baseline to $31.9 \pm 21.3$ following 6 months of treatment (-14.9 $\pm 16.5, p<0.0001$ ). The percentage of subjects with OSDI scores $>32$ (severe category) was reduced from $76 \%$ (38/50) at baseline to $44 \%$ (20/45) over the 6-month course of the study.

In the worse eye, there was no significant change in mean \pm SD TFBUT from baseline to 6 months $(-0.3 \pm 1.3$ seconds), and there was no significant change in the IBI from baseline to 6 months (-1.2 \pm 6.4 seconds). Similarly, there was no significant change from baseline to 6 months in the percentage area of tear deficiency/corneal exposure assessed using the OPI $2.0(-0.4 \pm 1.9)$.

Results of the Ora Calibra Ocular Discomfort Scale demonstrated significant improvements from baseline in both the worse eye $(-0.9 \pm 1.3, p<0.0001)$ and the fellow eye $(-0.7 \pm 1.4, p=0.0012)$. The Ora Calibra Ocular Discomfort and 4-Symptom Questionnaire also demonstrated significant improvements from baseline in ocular discomfort, as well as burning, dryness, grittiness, and stinging symptoms (Figure 3). A significant improvement was also 
Figure 1: Fluorescein staining in worse eye (A) and fellow eye (B), and lissamine green staining in worse eye (C) and fellow eye (D) at baseline and after 6 months of treatment with cyclosporine ophthalmic emulsion $0.05 \%$
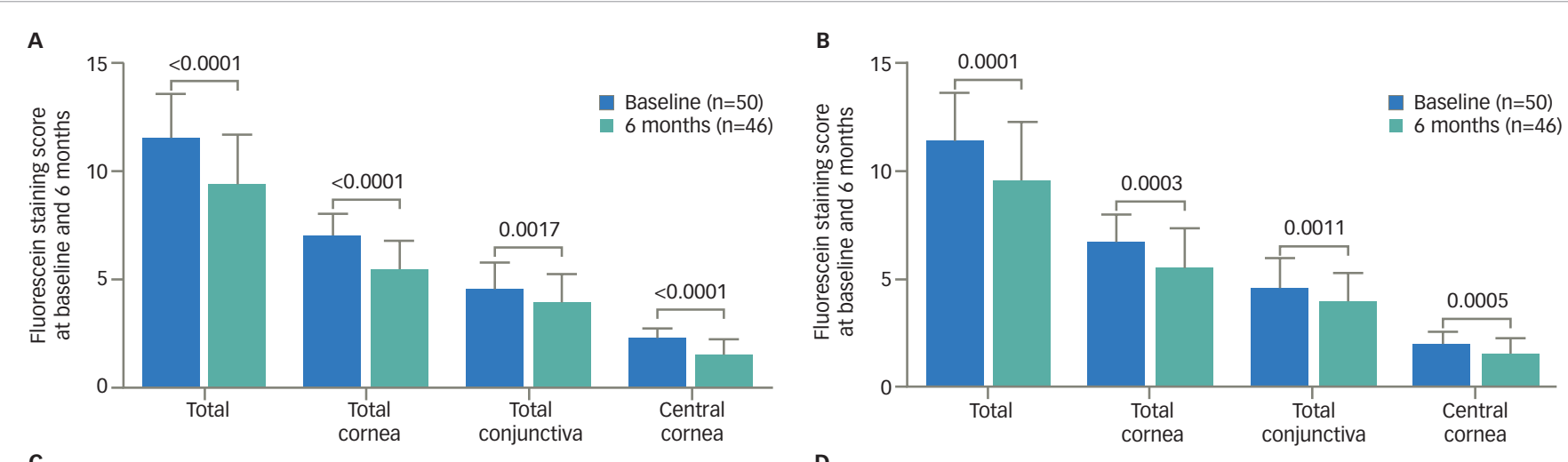

C

D
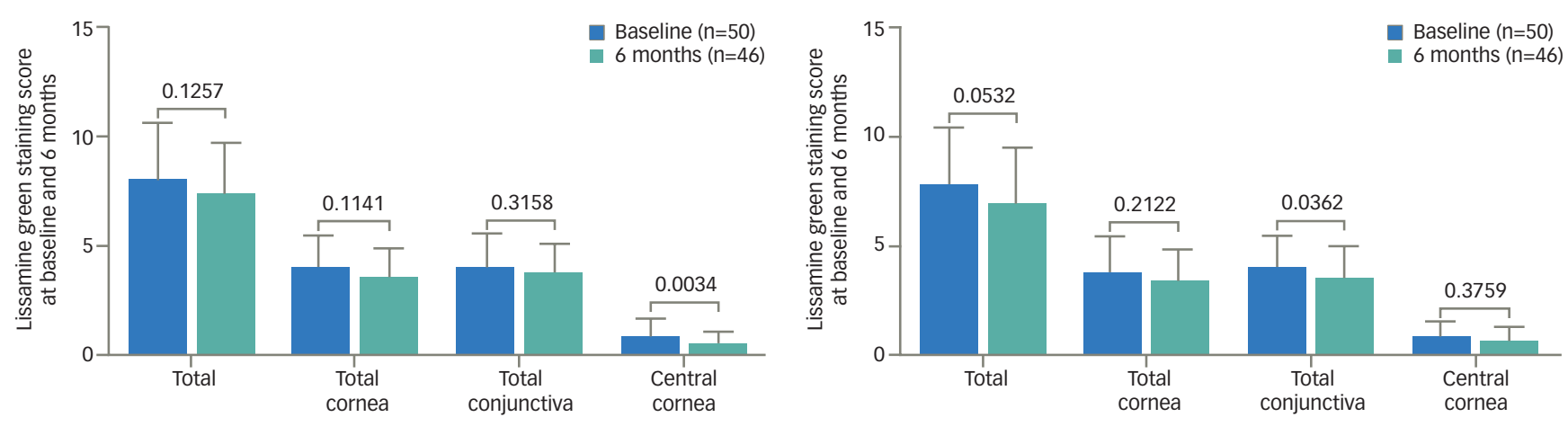

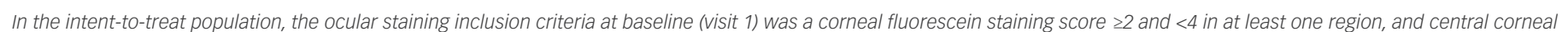

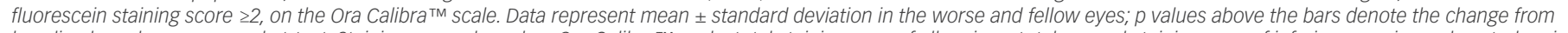

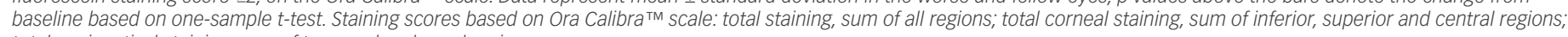
total conjunctival staining, sum of temporal and nasal regions.

Table 1: Reading test results at baseline, after 6 months of treatment with cyclosporine ophthalmic emulsion $0.05 \%$, and change from baseline in reading test results in the intent-to-treat population

\begin{tabular}{|c|c|c|c|c|c|c|c|c|c|}
\hline \multirow{2}{*}{$\begin{array}{l}\text { Test parameter } \\
\text { Mean } \pm \mathrm{SD}\end{array}$} & \multicolumn{3}{|l|}{ Baseline } & \multicolumn{3}{|l|}{ Month 6} & \multicolumn{3}{|c|}{ Change from baseline } \\
\hline & $\begin{array}{l}\text { Ora Calibra }^{\text {TM }} \\
\text { Menu Reading } \\
\text { Test }(n=50)\end{array}$ & $\begin{array}{l}\text { IReST } \\
\text { Reading } \\
\text { Test } \\
(n=50)\end{array}$ & $\begin{array}{l}\text { Wilkins Rate } \\
\text { of Reading } \\
\text { Test } \\
(n=50)\end{array}$ & $\begin{array}{l}\text { Ora Calibra }^{\text {TM }} \\
\text { Menu Reading } \\
\text { Test }(n=46)\end{array}$ & $\begin{array}{l}\text { IReST } \\
\text { Reading } \\
\text { Test } \\
(n=46)\end{array}$ & $\begin{array}{l}\text { Wilkins Rate } \\
\text { of Reading } \\
\text { Test } \\
(n=46)\end{array}$ & $\begin{array}{l}\text { Ora Calibra } \\
\text { Menu } \\
\text { Reading Test } \\
(n=46)\end{array}$ & $\begin{array}{l}\text { IREST } \\
\text { Reading } \\
\text { Test } \\
(n=46)\end{array}$ & $\begin{array}{l}\text { Wilkins Rate } \\
\text { of Reading } \\
\text { Test } \\
(n=46)\end{array}$ \\
\hline $\begin{array}{l}\text { Time to read } \\
\text { passage (seconds) }\end{array}$ & $42.4 \pm 18.4$ & $54.3 \pm 13.6$ & $126.0 \pm 28.6$ & $39.5 \pm 7.5$ & $53.4 \pm 13.4$ & $125.6 \pm 25.8$ & $\begin{array}{l}-3.0 \pm 14.8 \\
p=0.1808\end{array}$ & $\begin{array}{l}-1.2 \pm 8.4 \\
p=0.3372\end{array}$ & $\begin{array}{l}-0.7 \pm 24.5 \\
p=0.841\end{array}$ \\
\hline $\begin{array}{l}\text { Number of words } \\
\text { read incorrectly }\end{array}$ & $1.6 \pm 2.8$ & $3.2 \pm 5.5$ & $16.6 \pm 22.0$ & $1.9 \pm 3.0$ & $3.2 \pm 4.8$ & $12.5 \pm 16.9$ & $\begin{array}{l}0.3 \pm 1.8 \\
p=0.1946\end{array}$ & $\begin{array}{l}0.0 \pm 3.4 \\
p=1.0\end{array}$ & $\begin{array}{l}-4.1 \pm 16.6 \\
p=0.1043\end{array}$ \\
\hline Font size (points) & $12.0 \pm 0.0$ & $10.0 \pm 0.1$ & $9.0 \pm 0.0$ & $12.0 \pm 0.0$ & $10.0 \pm 0.0$ & $9.0 \pm 0.2$ & $\begin{array}{l}0.0 \pm 0.0 \\
p---\end{array}$ & $\begin{array}{l}0.0 \pm 0.2 \\
p=0.3227\end{array}$ & $\begin{array}{l}0.0 \pm 0.2 \\
p=0.1596\end{array}$ \\
\hline
\end{tabular}

$p$ values based on one-sample t-test.

IREST = international reading speed text; SD = standard deviation.

observed in ocular discomfort after the Wilkins Rate of Reading Test from 3.1 \pm 1.0 at baseline to $2.5 \pm 1.3(-0.6 \pm 1.2$-unit improvement, $p=0.0021)$ after 6 months of treatment. At study visits 2 and 4, diurnal symptoms (ocular discomfort and dry eye symptoms) were assessed in the morning and evening. At baseline, ocular discomfort and burning, dryness, and stinging symptoms were worse in the evening than in the morning, while grittiness showed no change between morning and evening assessments. After 6 months of treatment, ocular discomfort, burning, grittiness, and stinging symptoms all showed improvement in the evening and in the morning, but the magnitude of improvement was generally greater in the evening; some mild worsening in dryness was still observed in the evening compared with the morning (Table 2).

A significant increase in Schirmer's test score was observed in the worse eye from $7.6 \pm 5.5 \mathrm{~mm}$ at baseline to $11.4 \pm 8.6 \mathrm{~mm}$ after 6 months treatment with topical cyclosporine $(\mathrm{p}=0.0116)$. Similar results were observed in the 
Figure 2: Change from baseline in individual questions from the OSDI questionnaire after 6 months of treatment with cyclosporine ophthalmic emulsion $0.05 \%$, in the intent-to-treat population

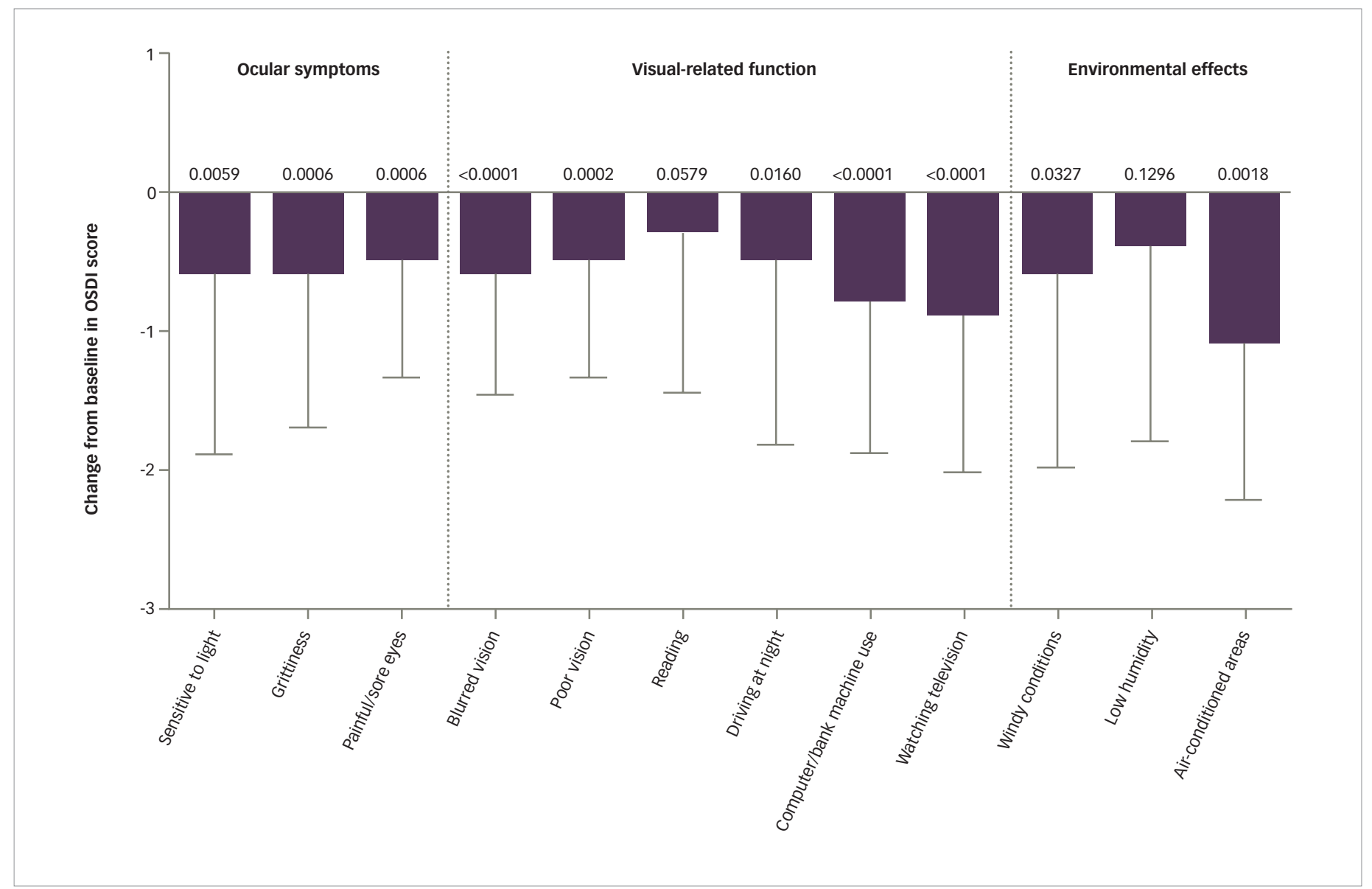

Data present mean \pm standard deviation in all subjects $(n=50) ; p$ values for the change from baseline based on one-sample $t$-test. OSDI, Ocular Surface Disease Index ${ }^{\circledR}$

fellow eye with an increase in Schirmer's score from $5.3 \pm 3.2 \mathrm{~mm}$ at baseline to $9.8 \pm 7.3 \mathrm{~mm}(\mathrm{p}<0.0001)$. Improvement from baseline of $\geq 10 \mathrm{~mm}$ was measured in $16 \%$ of worse eyes and fellow eyes, and improvements from baseline of $\geq 5 \mathrm{~mm}$ were measured in $32 \%$ of worse eyes and in $38 \%$ of fellow eyes after 6 months of treatment.

\section{Safety outcomes}

Twenty-two (44\%) subjects experienced a total of 36 treatment-emergent AEs, which included eight (16\%) subjects who experienced 12 ocular AEs (Table 3). Of the 12 ocular AEs, two were considered possibly related and one was considered definitely related to study drug. Among ocular AEs, reduced visual acuity occurred in three (6\%) subjects, and in each case was classified as mild and not related to study drug. Nasopharyngitis was the only non-ocular $A E$ that was reported in $\geq 5 \%$ of subjects $(n=3,6 \%$ ). Three serious non-ocular AEs were reported during the study (papillary thyroid cancer, peripheral vascular disorder, and carbon monoxide poisoning), none of which were considered to be related to treatment with cyclosporine ophthalmic emulsion $0.05 \%$. The subject diagnosed with papillary thyroid cancer was withdrawn from the study, whereas the other two completed the trial.

\section{Discussion}

The last 20 years have seen a rapid rise in the use of computers and handheld electronic devices, particularly among the younger population, where the use of such devices is typically frequent and prolonged. Excessive and prolonged use of video display terminals can exacerbate signs and symptoms of dry eye. ${ }^{30}$ Despite the increasing prevalence of dry eye among the young to middle aged (i.e., $<50$ years of age) and the impact on quality of life, ${ }^{31}$ dry eye is still often an underdiagnosed and poorly recognized ocular disease in this patient population.

This clinical study was designed to evaluate, in a real-world setting, the efficacy of cyclosporine ophthalmic emulsion $0.05 \%$ in dry eye subjects with a history of ocular discomfort and other dry eye symptoms while using video display terminals such as computers or hand-held devices. In this study, significant reductions in corneal and conjunctival staining were observed, consistent with prior studies of topical cyclosporine. ${ }^{20}$ In addition, significant improvements in visual function OSDI scores (blurry vision, poor vision, driving at night, working with computer or bank machine, and watching television) were also observed after 6 months of treatment. Similar findings have been reported previously in a single-center, 6-month, open-label study in $40 \mathrm{dry}$ eye subjects, who were significantly older (mean age 59 years, range 40-78) than the population in the present study (mean age 46 years, range 25-55). ${ }^{32}$

In addition to the reduction in overall staining, a significant reduction in fluorescein staining was also observed in the central corneal region, which may contribute to improvement in subject-rated visual function. ${ }^{33,34}$ 
Figure 3: Change from baseline in Ora Calibra ${ }^{\mathrm{TM}}$ Ocular Discomfort and 4-Symptom Questionnaire after 6 months of treatment with cyclosporine ophthalmic emulsion $0.05 \%$.

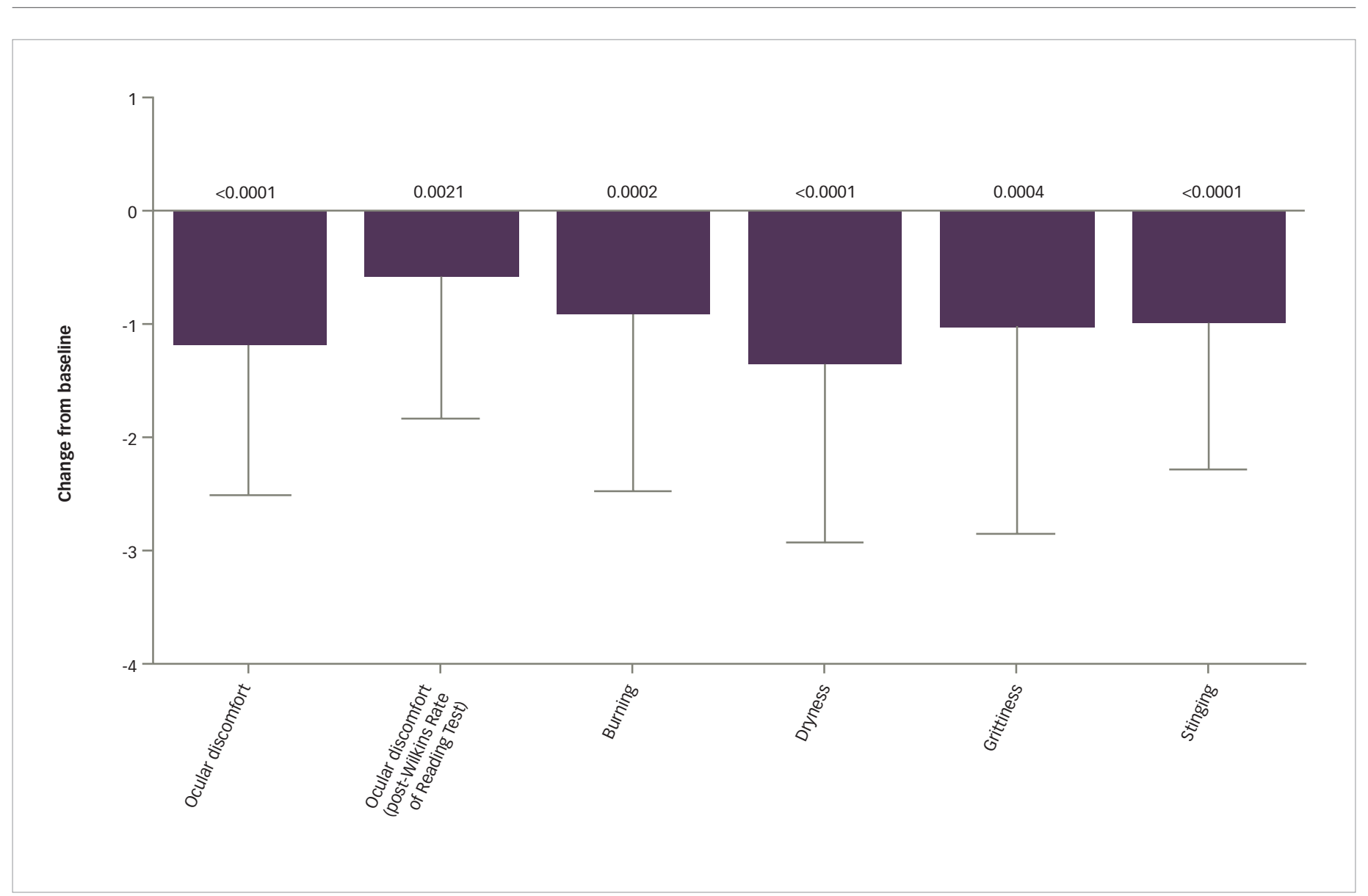

Data present mean \pm standard deviation in both eyes $(n=50) ; p$ values for the change from baseline based on one-sample $t$-test.

Table 2: Diurnal symptoms assessed in the morning and evening at baseline and after 6 months of treatment with cyclosporine ophthalmic emulsion $0.05 \%$, in the intent-to-treat population $(n=50)$

\begin{tabular}{|l|l|l|l|l|}
\hline \multirow{2}{*}{$\begin{array}{l}\text { Dry eye parameter } \\
\text { Mean } \pm \text { SD }\end{array}$} & \multicolumn{2}{l}{ Baseline study visit } & \multicolumn{2}{l}{ Month 6 study visit } \\
\cline { 2 - 5 } & Morning & Evening & Morning & Evening \\
\hline Ocular discomfort & $2.3 \pm 0.8$ & $2.6 \pm 1.1$ & $1.9 \pm 1.2$ & $1.7 \pm 1.3$ \\
\hline Burning & $1.5 \pm 1.2$ & $1.9 \pm 1.5$ & $1.0 \pm 1.3$ & $1.0 \pm 1.4$ \\
\hline Dryness & $2.9 \pm 1.1$ & $3.1 \pm 1.1$ & $2.2 \pm 1.5$ & $2.3 \pm 1.64$ \\
\hline Grittiness & $2.2 \pm 1.4$ & $2.2 \pm 1.6$ & $1.7 \pm 1.6$ & $1.6 \pm 1.4$ \\
\hline Stinging & $1.3 \pm 1.3$ & $1.5 \pm 1.5$ & $0.9 \pm 1.2$ & $0.8 \pm 1.2$ \\
\hline
\end{tabular}

Ocular discomfort assessed using the Ora Calibra ${ }^{\mathrm{TM}}$ Ocular Discomfort Scale $(0=$ none, $4=$ severe); burning/dryness/grittiness/stinging assessed using the Ora Calibra ${ }^{\text {тм }}$

4 Symptom Questionnaire ( $0=$ none, $5=$ worst).

$S D=$ standard deviation.

Significant reductions in conjunctival staining have also been reported in previously published studies investigating the use of topical cyclosporine in patients with dry eye. ${ }^{20,35,36}$ Similarly, as reported elsewhere, ${ }^{20,32,37,38}$ the use of cyclosporine has been associated with a significant increase in tear production assessed by Schirmer's test scores. In the present study, nearly half of the subjects (47.6\%) originally categorized with severe disease (OSDI
Table 3: Ocular adverse events reported during 6 months of treatment with cyclosporine ophthalmic emulsion $0.05 \%$

\begin{tabular}{|l|l|}
\hline Adverse event & Subjects, $\mathrm{n}(\%)$ \\
\hline Total number of ocular adverse events & $8(16)$ \\
\hline Visual acuity reduced & $3(6)$ \\
\hline Eyelid pain & $1(2)$ \\
\hline Blepharospasm & $1(2)$ \\
\hline Episcleritis & $1(2)$ \\
\hline Eye pruritus & $1(2)$ \\
\hline Infections and infestations & $1(2)$ \\
\hline Instillation site pain & $1(2)$ \\
\hline Lacrimation increased & $1(2)$ \\
\hline Vision blurred & $1(2)$ \\
\hline
\end{tabular}

score $>32$ ) at baseline improved by at least one category in dry eye disease severity after 6 months of treatment. Perry et al. previously reported similar findings where the use of topical cyclosporine led to improvements in OSDI scores in 158 patients with mild, moderate, or severe dry eye disease. ${ }^{36}$ Cyclosporine ophthalmic emulsion $0.05 \%$ was well tolerated in the study, consistent with its established safety profile. ${ }^{20,21,32,35}$ 
Visual functioning assessed using the Ora Calibra Menu Reading Test, IReST Reading Test, and the Wilkins Rate of Reading Test showed improvements following treatment with topical cyclosporine, although differences in time to read passages and the number of words read incorrectly did not reach statistical significance. OSDI visual function scores did show significant improvement (reduction) from baseline. No studies have been conducted correlating reading test results and subject-rated visual function. Our study demonstrates improvement in both assessments, suggesting subjects experienced benefit in vision-related outcomes following 6 months of treatment with topical cyclosporine.

Dry eye disease is often associated with symptoms that vary throughout the day, often leading to difficulties in evening visual activities such as reading, television or computer use. Over the course of a day, the combination of environmental exposure and visual tasking activities may have a cumulative effect on the ocular surface resulting in a measurable pattern of progressive increase in signs and symptoms.,39 Treatment with topical cyclosporine demonstrated significant reduction in diurnal symptoms assessed in this study. After 6 months, subjects demonstrated improvements in ocular discomfort, burning, grittiness, and stinging symptoms in both the evening and the morning, with even more robust reductions seen in evening symptoms relative to baseline.

Treatment with cyclosporine ophthalmic emulsion resulted in significant symptom relief. Significant improvements in symptoms measured using the Ora Calibra Ocular Discomfort Scale and Ora Calibra Ocular Discomfort and 4-Symptom Questionnaire were observed following 6 months of treatment. In addition, ocular discomfort scores collected following Wilkins Rate of Reading Tests were significantly reduced in this study. In the pivotal phase
III trials involving 877 patients, significant improvements from baseline were observed in dryness, sandy/gritty feeling, burning, and stinging symptoms following the use of cyclosporine. ${ }^{20}$ Using the Ora Calibra Ocular Discomfort and 4-Symptom Questionnaire, significant improvements in ocular discomfort and dry eye symptoms have also been reported following cyclosporine instillation in the single-center, 6-month, open-label study. ${ }^{32}$

The lack of a comparator or control group could potentially be a study limitation. However, within-subject comparisons often provide the most reliable data for conditions such as dry eye disease, which has a highly variable presentation of signs and symptoms due to differences in exogenous factors that impact each subject. Variations in environmental conditions and disease phenotype across subjects can create significant disparities in the spectrum of individual dry eye pathophysiology. The lack of a placebo/control group does preclude attributing any of the AEs reported in this study to the effects of cyclosporine ophthalmic emulsion $0.05 \%$. In addition, the reading tests used were likely too short to show a change in reading speed in patients with normal vision and dry eye disease. Although there was improvement in corneal staining which translated to significant subjective improvement in visual function, this did not translate into faster reading.

\section{Conclusion}

Although dry eye disease is often considered to affect the elderly, a growing body of literature has confirmed the rising prevalence of dry eye in the younger adult population. This study demonstrates the efficacy and safety of cyclosporine ophthalmic emulsion in treating a younger dry eye population who experience ocular discomfort when engaging in visual tasking. Following 6 months of treatment with cyclosporine ophthalmic emulsion, significant improvements were observed in ocular signs, symptoms, and subject-rated visual function. $\square$
1. Craig JP, Nichols KK, AKpek EK, et al. TFOS DEWS II definition and classification report Ocul Surf. 2017:15:276-83.

2. Stevenson W, Chauhan SK, Dana R. Dry eye disease: An immune-mediated ocular surface disorder. Arch Ophthalmol. 2012;130:90-100

3. Pflugfelder SC, Corrales RM, de Paiva CS. T helper cytokines in dry eye disease. Exp Eye Res. 2013;117:118-25.

4. Stern ME, Schaumburg CS, Pflugfelder SC. Dry eye as a mucosal autoimmune disease. Int Rev Immunol. 2013;32:19-41.

5. Walker PM, Lane KJ, Ousler III GW, Abelson MB. Diurnal variation of visual function and the signs and symptoms of dry eye. Cornea. 2010:29:607-12.

6. Uchino M, Schaumberg DA. Dry eye disease: Impact on quality of life and vision. Curr Ophthalmol Rep. 2013;1:51-7.

7. Mertzanis P, AbetZ L, Rajagopalan K, et al. The relative burden of dry eye in patients' lives: Comparisons to a U.S. normative sample. Invest Ophthalmol Vis Sci. 2005;46:46-50.

8. Miljanović B, Dana R, Sullivan DA, Schaumberg DA. Impact of dry eye syndrome on vision-related quality of life. Am J Ophthalmol. 2007:143:409-15.

9. MCDonald M, Patel DA, Keith MS, Snedecor SJ. Economic and humanistic burden of dry eye disease in Europe, North America, and Asia: A systematic literature review. Ocul Surf. 2016;14:144-67.

10. Schiffman RM, Christianson MD, Jacobsen G, Hirsch JD, Reis BL. Reliability and validity of the Ocular Surface Disease Index. Arch Ophthalmol. 2000;118:615-21.

11. Stapleton F, Alves M, Bunya VY, et al. TFOS DEWS II epidemiology report. Ocul Surf. 2017;15:334-65.

12. MOSS SE, Klein R, Klein BE. Prevalence of and risk factors for dry eye syndrome. Arch Ophthalmol. 2000;118:1264-8

13. Schaumberg DA, Sullivan DA, Buring JE, Dana MR. Prevalence of dry eye syndrome among US women. Am I Ophthalmol. 2003;136:318-26.

14. Sweeney DF, Millar TJ, Raju SR. Tear film stability: A review. Exp Eye Res. 2013;117:28-38.

15. Courtin R, Pereira B, Naughton G, et al. Prevalence of dry eye disease in visual display terminal workers: A systematic review and meta-analysis. BMJ Open. 2016;6:e009675.

16. Vitale S, Goodman LA, Reed GF, Smith JA. Comparison of the NEI-VFQ and OSDI questionnaires in patients with Sjögren's syndrome-related dry eye. Health Qual Life Outcomes. 2004;2:44.
17. Sullivan BD, Crews $L A$, Messmer EM, et al. Correlations between commonly used objective signs and symptoms for the diagnosis of dry eye disease: Clinical implications. Acta Ophthalmol. 2014;92:161-6.

18. Pult H, Purslow C, Murphy PJ. The relationship between clinical signs and dry eye symptoms. Eye. 2011;25:502-10.

19. Allergan plc. RESTASIS ${ }^{\circledast}$ (cyclosporine ophthalmic emulsion) 0.05\%, prescribing information. 2017. Available at: https://Www allergan.com/assets/pdf/restasis pi.pdf (accessed November 22, 2019).

20. Sall K, Stevenson OD, Mundorf TK, Reis BL. Two multicenter, randomized studies of the efficacy and safety of cyclosporine ophthalmic emulsion in moderate to severe dry eye disease. Ophthalmology. 2000;107:631-9.

21. Stevenson D, Tauber J, Reis BL, The Cyclosporin A Phase 2 Study Group. Efficacy and safety of cyclosporin A ophthalmic emulsion in the treatment of moderate-to-severe dry eye disease: A dose-ranging, randomized trial. Ophthalmology. 2000:107:967-74.

22. Shire US Inc. XIIDRA TM (lifitegrast ophthalmic solution) $5 \%$, full prescribing information. 2016. Available at: http://www. shirecontent.com/PI/PDFS/XIIDRA_USA_ENG.pdf (accessed November 22, 2019)

23. Lane KJ, Ousler III GW, Rodriguez JD, Angjeli E. Continuous blink recording and the relationship of diurnal blink parameters to diurnal change in signs and symptoms of dry eye [abstract]. Invest Ophthalmol Vis Sci. 2015:56:4447.

24. Ousler III GW, Devries DK, Karpecki PM, Ciolino JB. An evaluation of Retaine ${ }^{\mathrm{TM}}$ ophthalmic emulsion in the management of tear film stability and ocular surface staining in patients diagnosed with dry eye. Clin Ophthalmol. 2015;9:235-43.

25. Semba CP, Torkildsen GL, Lonsdale JD, et al. A phase 2 randomized, double-masked, placebo-controlled study of a novel integrin antagonist (SAR 1118) for the treatment of dry eye. Am J Ophthalmol. 2012;153:1050-60.

26. Ozcura F, Aydin $\mathrm{S}$, Helvaci MR. Ocular surface disease index for the diagnosis of dry eye syndrome. Ocul Immunol Inflamm. 2007;15:389-93.

27. Miller KL, Walt JG, Mink DR, et al. Minimal clinically important difference for the Ocular Surface Disease Index. Arch Ophthalmol 2010;128:94-101.
28. Abelson R, Lane KJ, Rodriguez J, et al. Validation and verification of the OPI 2.0 System. Clin Ophthalmol. 2012;6:613-22.

29. Grosskreutz CL, Hockey HU, Serra D, Dryja TP. Dry eye signs and symptoms persist during systemic neutralization of IL-1 $\beta$ by canakinumab or IL-17a by secukinumab. Cornea. 2015;34:1551-6.

30. Acosta MC, Gallar J, Belmonte C. The influence of eye solutions on blinking and ocular comfort at rest and during work at video display terminals. Exp Eye Res. 1999;68:663-9.

31. Paulsen AJ, Cruickshanks KJ, Fischer ME, et al. Dry eye in the Beaver Dam Offspring Study: Prevalence, risk factors, and healthrelated quality of life. Am J Ophthalmol. 2014;157:799-806.

32. Stonecipher KG, Torkildsen GL, Ousler III GW, Morris S, Villanueva L, Hollander DA. The IMPACT study: A prospective evaluation of the effects of cyclosporine ophthalmic emulsion $0.05 \%$ on ocular surface staining and visual performance in patients with dry eye. Clin Ophthalmol. 2016;10:887-95.

33. Lemp MA. Advances in understanding and managing dry eye disease. Am J Ophthalmol. 2008:146:350-56.

34. Adatia FA, Michaeli-Cohen A, Naor J, Caffery B, Bookman A, Slomovic A. Correlation between corneal sensitivity, subjective dry eye symptoms and corneal staining in Sjögren's syndrome. Can J Ophthalmol. 2004;39:767-71.

35. Straub M, Bron AM, Muselier-Mathieu A, Creuzot-Garcher C. Long-term outcome after topical ciclosporin in severe dry eye disease with a 10-year follow-up. Br I Ophthalmol. 2016:100:1547-50

36. Perry $H D$, Solomon R, Donnenfeld ED, et al. Evaluation of topical cyclosporine for the treatment of dry eye disease. Arch Ophthalmol. 2008;126:1046-50

37. Roberts CW, Carniglia PE, Brazzo BG. Comparison of topical cyclosporine, punctal occlusion, and a combination for the treatment of dry eye. Cornea. 2007;26:805-9.

38. Guzey M, Karaman SK, Satici A, Ozardali I, Sezer S, Bozkurt O Efficacy of topical cyclosporine $A$ in the treatment of severe trachomatous dry eye. Clin Exp Ophthalmol. 2009:37:541-9.

39. Barabino S, Labetoulle M, Rolando M, Messmer EM. Understanding symptoms and quality of life in patients with $\mathrm{dry}$ eye syndrome. Ocul Surf. 2016;14:365-76.

40. Ding J, Sullivan DA. Aging and dry eye disease. Exp Gerontol. 2012:47:483-90. 


\section{Appendix 1}

Ocular Surface Disease Index ${ }^{\odot}(\mathrm{OSDI})^{\odot}$ Scoring Chart

\section{OCULAR SURFACE DISEASE INDEX ${ }^{\odot}$}

Please answer the following questions by checking the box that best represents your answer.

Have you experienced any of the following during the last week:

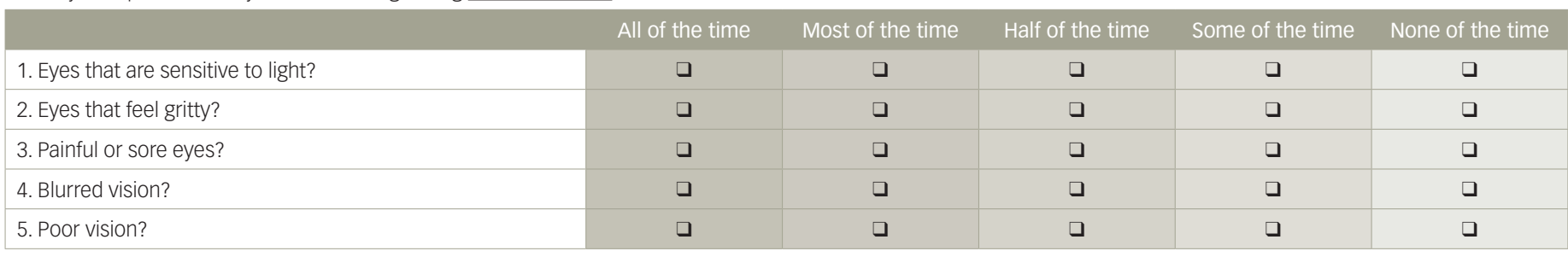

Have problems with your eyes limited you in performing any of the following during the last week:

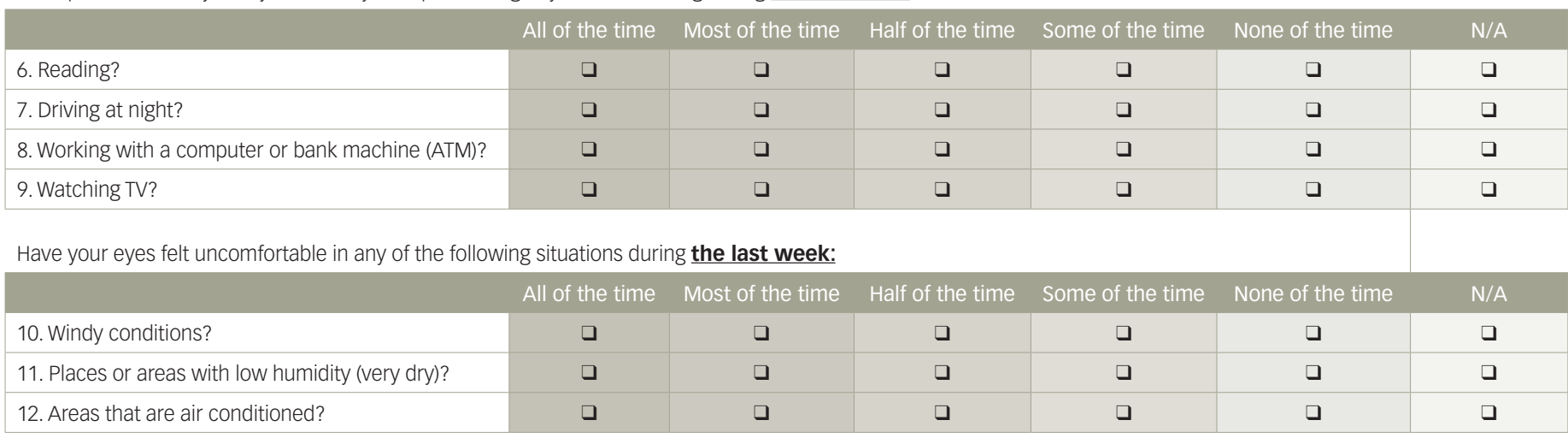

Scoring Instructions

\section{Item Scoring}

The total OSDI score is calculated based on the following formula:

$\mathrm{OSDI}=\frac{(\text { sum of severity for all questions answered }) \times(100)}{(\text { total } \# \text { of questions answered }) \times(4)}$

where the severity was graded on a scale of
\[ \begin{array}{l}0=\text { none of the time. } \\ 1=\text { some of the time. } \\ 2=\text { half of the time. } \\ 3=\text { most of the time. } \\ 4=\text { all of the time. }\end{array} \]

\section{Interpretation}

A score of 100 corresponds to complete disability (a response of "all of the time" to all questions answered), while a score of 0 corresponds to no disability (a response of "none of the time" to all questions answered). Therefore, change from baseline of -12.5 corresponds to an improvement by at least one category in half of the questions answered.

\section{Subscale Scoring}

Subscales scores are computed similarly with only the questions from each subscale used to generate its own score. Therefore, any subscales analyzed separately would also have a maximum possible score of 100 .

The three subscales (vision-related function, ocular symptoms and environmental triggers) are broken out as follows:

\begin{tabular}{|l|l|}
\hline Subscale & Questions \\
\hline Vision-Related Function & $4,5,6,7,8,9$ \\
\hline Ocular Symptoms & $1,2,3$ \\
\hline Environmental Triggers & $10,11,12$ \\
\hline
\end{tabular}

